\title{
Energy-Momentum Tensor in Electromagnetic Theory and Gravitation from Relativistic Quantum Equations
}

\author{
Valeriy Dvoeglazov* \\ UAF, Universidad de Zacatecas \\ E-mail: vdvoeglazoveyahoo.com.mx
}

Recently, several discussions on the possible observability of 4-vector fields have been published in literature. Furthermore, several authors recently claimed existence of the helicity $=0$ fundamental field. We re-examine the theory of antisymmetric tensor fields and 4-vector potentials. We study the massless limits. In fact, theoretical motivation for this venture is the old papers of Ogievetskiı and Polubarinov, Hayashi, and Kalb and Ramond. They proposed the concept of the notoph, whose helicity properties are complementary to those of the photon. We analyze the quantum field theory with taking into account mass dimensions of the notoph and the photon. We also proceed to derive equations for the symmetric tensor of the second rank on the basis of the Bargmann-Wigner formalism. They are consistent with the general relativity. Particular attention has been paid to the correct definitions of the energy-momentum tensor and other Nöther currents. We estimate possible interactions, fermion-notoph, graviton-notoph, photon-notoph.

PACS number: 03.65.Pm , 04.50.-h, 11.30.Cp

Frontiers of Fundamental Physics 14

15-18 July 2014

Aix Marseille University (AMU) Saint-Charles Campus, Marseille, France

\footnotetext{
* Speaker.
} 
In this presentation we re-examine the theory of the 4-vector field, the antisymmetric tensor fields of the second ranks and the spin-2 fields coming from the modified Bargmann-Wigner formalism. In the series of the papers $[1,2,3,4]$ we tried to find connection between the theory of the quantized antisymmetric tensor (AST) field of the second rank (and that of the corresponding 4-vector field) with the $2(2 s+1)$ Weinberg-Tucker-Hammer formalism [5, 6]. Several previously published works $[7,8,9]$ introduced the concept of the notoph (the Kalb-Ramond field) which is constructed on the basis of the antisymmetric tensor "potentials", cf. [10, 11, 12, 13]. It represents itself the non-trivial spin-0 field. We posed the problems, whether the massless quantized AST potential and the quantized 4-vector field are transverse or longitudinal fields (in the sense if the helicity $h= \pm 1$ or $h=0)$ ? can the electromagnetic potential be a 4-vector in a quantized theory? contradictions with the Weinberg theorem "that no symmetric tensor field of rank $s$ can be constructed from the creation and annihilation operators of massless particles of spin $s$ "? how should the massless limit be taken?

First of all, we note that 1) "...In natural units $(c=\hbar=1) \ldots$ a lagrangian density, since the action is dimensionless, has dimension of [energy $]^{4 " ;}$ 2) One can always renormalize the lagrangian density and "one can obtain the same equations of motion... by substituting $L \rightarrow\left(1 / M^{N}\right) L$, where $M$ is an arbitrary energy scale", cf. [3]; 3) the right physical dimension of the field strength tensor $F^{\mu v}$ is [energy] ; " "the transformation $F^{\mu v} \rightarrow(1 / 2 m) F^{\mu v}$ [which was regarded in Ref. [14, 15]] ... requires a more detailed study ... [because] the transformation above changes its physical dimension: it is not a simple normalization transformation". Furthermore, in the first papers on the notoph the authors used the normalization of the 4-vector $F^{\mu}$ field, which is related to a third-rank antisymmetric field tensor, to [energy $]^{2}$ and, hence, the antisymmetric tensor "potentials" $A^{\mu \nu}$, to [energy] ${ }^{1}$. We discuss these problems on the basis of the generalized Bargmann-Wigner formalism [16, 17]. The Proca, Maxwell and Einstein formalisms are generalized, Ref. [4]. A field of the rest mass $m$ and the spin $s \geq \frac{1}{2}$ is represented by a completely symmetric multispinor of rank $2 s$. The particular cases $s=1$ and $s=\frac{3}{2}$ have been considered in the textbooks, e. g., Ref. [17]. Nevertheless, questions of the redundant components of the higher-spin relativistic equations are not yet understood in detail [18]. The questions of quantization, interactions and relations between various higher-spin theories have also been discussed.

The Bargmann-Wigner postulates lead to the Kemmer set of the $s=0$ equations after algebraic transformations

$$
m \phi=0, \quad m \widetilde{\phi}=-i \partial_{\mu} \widetilde{A}^{\mu}, \quad m \widetilde{A}^{\mu}=-i \partial^{\mu} \widetilde{\phi}
$$

For the $s=1$ case they lead to the Proca-Duffin-Kemmer set:

$$
\partial_{\alpha} F^{\alpha \mu}+\frac{m}{2} A^{\mu}=0, \quad 2 m F^{\mu v}=\partial^{\mu} A^{v}-\partial^{v} A^{\mu} .
$$

The dual antisymmetric tensor can also be used [15]. In the meantime, the textbooks equations are obtained from (2) after the normalization change $A_{\mu} \rightarrow 2 m A_{\mu}$ or $F_{\mu \nu} \rightarrow \frac{1}{2 m} F_{\mu \nu}$.

In order to be able to answer the question about the behaviour of eigenvalues of the spin operator $\mathbf{J}^{i}=\frac{1}{2} \varepsilon^{i j k} J^{j k}$ in the massless limit one should know the behaviour of the fields $F_{\mu \nu}$ and/or $A_{\mu}$ in the massless limit. We choose the usual definitions (p. 209 of [19]) for polarization vectors 
$\varepsilon^{\mu}(\mathbf{0}, \sigma):$

$$
\varepsilon^{\mu}(\mathbf{0},+1)=-\frac{1}{\sqrt{2}}\left(\begin{array}{c}
0 \\
1 \\
i \\
0
\end{array}\right), \varepsilon^{\mu}(\mathbf{0}, 0)=\left(\begin{array}{l}
0 \\
0 \\
0 \\
1
\end{array}\right), \varepsilon^{\mu}(\mathbf{0},-1)=\frac{1}{\sqrt{2}}\left(\begin{array}{c}
0 \\
1 \\
-i \\
0
\end{array}\right)
$$

and boost them to an arbitrary frame $\left(\widehat{p}_{i}=p_{i} /|\mathbf{p}|, \gamma=E_{p} / m\right)$, p. 68 of Ref. [19],

$$
\begin{aligned}
& \varepsilon^{\mu}(\mathbf{p}, \sigma)=L^{\mu} \quad{ }_{v}(\mathbf{p}) \varepsilon^{v}(\mathbf{0}, \sigma) \\
& L^{0} \quad{ }_{0}(\mathbf{p})=\gamma, \quad L^{i} \quad{ }_{0}(\mathbf{p})=L^{0} \quad{ }_{i}(\mathbf{p})=\widehat{p}_{i} \sqrt{\gamma^{2}-1} \\
& L^{i} \quad{ }_{k}(\mathbf{p})=\delta_{i k}+(\gamma-1) \widehat{p}_{i} \widehat{p}_{k}
\end{aligned}
$$

The normalization of the field functions in the momentum representation is thus chosen to the negative unit, $\varepsilon_{\mu}^{*}(\mathbf{p}, \sigma) \varepsilon^{\mu}(\mathbf{p}, \sigma)=-1$. We observe that in the massless limit all the defined polarization vectors of the momentum space do not have good behaviour; the functions describing spin-1 particles tend to infinity. Nevertheless, after renormalizing the polarization vectors, e. g., $\varepsilon^{\mu} \rightarrow u^{\mu} \equiv m \varepsilon^{\mu}$ we come to the field functions in the momentum representation:

$$
\begin{gathered}
u^{\mu}(\mathbf{p},+1)=-\frac{N}{\sqrt{2} m}\left(\begin{array}{c}
p_{r} \\
m+\frac{p_{1} p_{r}}{E_{p}+m} \\
i m+\frac{p_{2} p_{r}}{E_{p}+m} \\
\frac{p_{3} p_{r}}{E_{p}+m}
\end{array}\right), \quad u^{\mu}(\mathbf{p},-1)=\frac{N}{\sqrt{2} m}\left(\begin{array}{c}
p_{l} \\
m+\frac{p_{1} p_{l}}{E_{p}+m} \\
-i m+\frac{p_{2} p_{l}}{E_{p}+m} \\
\frac{p_{3} p_{l}}{E_{p}+m}
\end{array}\right), \\
u^{\mu}(\mathbf{p}, 0)=\frac{N}{m}\left(\begin{array}{c}
p_{3} \\
\frac{p_{1} p_{3}}{E_{p}+m} \\
\frac{p_{2} p_{3}}{E_{p}+m} \\
m+\frac{p_{3}^{2}}{E_{p}+m}
\end{array}\right),
\end{gathered}
$$

( $N=m$ and $\left.p_{r, l}=p_{1} \pm i p_{2}\right)$ which do not diverge in the massless limit. Two of the massless functions (with $\sigma= \pm 1$ ) are equal to zero when a particle, described by this field, is moving along the third axis $\left(p_{1}=p_{2}=0, p_{3} \neq 0\right)$. The third one $(\sigma=0)$ is

$$
\left.u^{\mu}\left(p_{3}, 0\right)\right|_{m \rightarrow 0}=\left(\begin{array}{c}
p_{3} \\
0 \\
0 \\
\frac{p_{3}^{2}}{E_{p}}
\end{array}\right) \equiv\left(\begin{array}{c} 
\pm E_{p} \\
0 \\
0 \\
E_{p}
\end{array}\right)
$$

and at the rest $\left(E_{p}=p_{3} \rightarrow 0\right)$ also vanishes. Thus, an appropriate field operator describes the "longitudinal photons" what is in the complete accordance with the Weinberg theorem $B-A=h$ for massless particles (we use the $D(1 / 2,1 / 2)$ representation). The change of the normalization can lead to the change of physical content described by the classical field. In the quantum case one should somehow fix the form of commutation relations by some physical principles. Furthermore, the properties of the physical fields $F^{\mu v}$ are obtained from $\mathbf{B}^{(+)}(\mathbf{p}, \sigma)=\frac{i}{2 m} \mathbf{p} \times \mathbf{u}(\mathbf{p}, \sigma)$, $\mathbf{E}^{(+)}(\mathbf{p}, \sigma)=\frac{i}{2 m} p_{0} \mathbf{u}(\mathbf{p}, \sigma)-\frac{i}{2 m} \mathbf{p} u^{0}(\mathbf{p}, \sigma)$ in the massless zero-momentum limit. For the sake of 
completeness let us present the vector corresponding to the "time-like" polarization:

$$
u^{\mu}\left(\mathbf{p}, 0_{t}\right)=\frac{N}{m}\left(\begin{array}{c}
E_{p} \\
p_{1} \\
p_{2} \\
p_{3}
\end{array}\right) \quad, \quad \mathbf{B}^{( \pm)}\left(\mathbf{p}, 0_{t}\right)=\mathbf{0} \quad, \quad \mathbf{E}^{( \pm)}\left(\mathbf{p}, 0_{t}\right)=\mathbf{0} .
$$

The polarization vector $u^{\mu}\left(\mathbf{p}, 0_{t}\right)$ has good behaviour in $m \rightarrow 0, N=m$ (and also in the subsequent limit $\mathbf{p} \rightarrow \mathbf{0}$ ) and it may correspond to some field (particle). As one can see the field operator composed of the state of longitudinal polazrizations (e.g., as the "positive-energy" solution) and that of time-like polarization (e.g., as the "negative-energy" solution) may describe a situation when a particle and an antiparticle have opposite intrinsic parities.

The Lagrangian may include, in general, mass term:

$$
\begin{aligned}
& L=\frac{1}{4}\left(\partial_{\mu} A_{v \alpha}\right)\left(\partial^{\mu} A^{v \alpha}\right)-\frac{1}{2}\left(\partial_{\mu} A^{\mu \alpha}\right)\left(\partial^{v} A_{v \alpha}\right)-\frac{1}{2}\left(\partial_{\mu} A_{v \alpha}\right)\left(\partial^{v} A^{\mu \alpha}\right) \\
& +\frac{1}{4} m^{2} A_{\mu v} A^{\mu \nu} .
\end{aligned}
$$

Here we use the notation $A_{\mu \nu}$ for the AST due to possible different "mass dimensions" of the fields. The massless $(m=0)$ Lagrangian is connected with the Lagrangians used in other theories by adding the total derivative:

$$
L_{C F T}=L+\frac{1}{2} \partial_{\mu}\left(A_{v \alpha} \partial^{v} A^{\mu \alpha}-A^{\mu \alpha} \partial^{v} A_{v \alpha}\right) .
$$

The Kalb-Ramond gauge-invariant form (with respect to the "gauge" transformations $A_{\mu \nu} \rightarrow A_{\mu \nu}+$ $\left.\partial_{v} \Lambda_{\mu}-\partial_{\mu} \Lambda_{v}\right)$, Ref. [7, 8, 9], is obtained only if one uses the Fermi procedure mutatis mutandis by removing the additional "phase" field $\lambda\left(\partial_{\mu} A^{\mu v}\right)^{2}$ from the Lagrangian. This has certain analogy with the QED, where the question, whether the Lagrangian is gauge-invariant or not, is solved depending on the presence of the term $\lambda\left(\partial_{\mu} A^{\mu}\right)^{2}$. The Lagrangian leads to the equation of motion in the following form:

$$
\frac{1}{2}\left(\square+m^{2}\right) A_{\mu v}+\left(\partial_{\mu} A_{\alpha v^{\alpha}}{ }^{\alpha}-\partial_{v} A_{\alpha \mu}{ }^{\alpha}\right)=0
$$

It is this equation for antisymmetric-tensor-field components that follows from the Proca-DuffinKemmer consideration provided that $m \neq 0$ and in the final expression one takes into account the Klein-Gordon equation.

Following the variation procedure one can obtain the energy-momentum tensor:

$$
\Theta^{\lambda \beta}=\frac{1}{2}\left[\left(\partial^{\lambda} A_{\mu \alpha}\right)\left(\partial^{\beta} A^{\mu \alpha}\right)-2\left(\partial_{\mu} A^{\mu \alpha}\right)\left(\partial^{\beta} A_{\alpha}^{\lambda}\right)-2\left(\partial^{\mu} A^{\lambda \alpha}\right)\left(\partial^{\beta} A_{\mu \alpha}\right)\right]-L g^{\lambda \beta} .
$$

One can also obtain that for rotations the corresponding variation of the wave function is found from the formula: $\delta A^{\alpha \beta}=\frac{1}{2} \omega^{\kappa \tau} T_{\kappa \tau}^{\alpha \beta, \mu v} A_{\mu v}$. The generators of infinitesimal transformations are defined as

$$
\begin{aligned}
& T_{\kappa \tau}^{\alpha \beta, \mu \nu}=\frac{1}{2} g^{\alpha \mu}\left(\delta_{\kappa}^{\beta} \delta_{\tau}^{v}-\delta_{\tau}^{\beta} \delta_{\kappa}^{v}\right)+\frac{1}{2} g^{\beta \mu}\left(\delta_{\kappa}^{v} \delta_{\tau}^{\alpha}-\delta_{\tau}^{v} \delta_{\kappa}^{\alpha}\right)+ \\
& +\frac{1}{2} g^{\alpha v}\left(\delta_{\kappa}^{\mu} \delta_{\tau}^{\beta}-\delta_{\tau}^{\mu} \delta_{\kappa}^{\beta}\right)+\frac{1}{2} g^{\beta v}\left(\delta_{\kappa}^{\alpha} \delta_{\tau}^{\mu}-\delta_{\tau}^{\alpha} \delta_{\kappa}^{\mu}\right)
\end{aligned}
$$


Thus,

$$
J_{\kappa \tau}=\int d^{3} \mathbf{x}\left[\frac{\partial L}{\partial\left(\partial A^{\alpha \beta} / \partial t\right)} T_{\kappa \tau}^{\alpha \beta, \mu v} A_{\mu v}\right]
$$

and

$$
\begin{aligned}
& J_{\kappa \tau}=\int d^{3} \mathbf{x}\left[\left(\partial_{\mu} A^{\mu v}\right)\left(g_{0 \kappa} A_{v \tau}-g_{0 \tau} A_{v \kappa}\right)-\left(\partial_{\mu} A^{\mu}{ }_{\kappa}\right) A_{0 \tau}+\left(\partial_{\mu} A_{\tau}^{\mu}\right) A_{0 \kappa}+\right. \\
& \left.+A^{\mu}{ }_{\kappa}\left(\partial_{0} A_{\tau \mu}+\partial_{\mu} A_{0 \tau}+\partial_{\tau} A_{\mu 0}\right)-A_{\tau}^{\mu}{ }_{\tau}\left(\partial_{0} A_{\kappa \mu}+\partial_{\mu} A_{0 \kappa}+\partial_{\kappa} A_{\mu 0}\right)\right]
\end{aligned}
$$

Furthermore, one should choose the space-like normalized vector $n^{\mu} n_{\mu}=-1$ and find the explicit form of the relativistic spin after lengthy calculations:

$$
\begin{aligned}
& \left(W_{\mu} \cdot n^{\mu}\right)=-(\mathbf{W} \cdot \mathbf{n})=-\frac{1}{2} \varepsilon^{i j k} n^{k} J^{i j} p^{0} \\
& \mathbf{J}^{k}=\varepsilon^{i j k} \int d^{3} \mathbf{x}\left[A^{0 i}\left(\partial_{\mu} A^{\mu j}\right)+A_{\mu}^{j}\left(\partial^{0} A^{\mu i}+\partial^{\mu} A^{i 0}+\partial^{i} A^{0 \mu}\right)\right]
\end{aligned}
$$

Now it becomes obvious that the application of the generalized Lorentz conditions leads in such a formulation to the fact that the resulting Kalb-Ramond field is longitudinal (helicity $h=0$ ). All the components of the angular momentum tensor for this case are identically equated to zero.

According to $[7$, Eqs. $(9,10)]$ we proceed in the construction of the "potentials" for the notoph (by taking, in fact, the 4-cross product of polarization vectors) $\tilde{F}_{\mu \nu}(\mathbf{p}) \sim A_{\mu \nu}(\mathbf{p})=N\left[\varepsilon_{\mu}^{(1)}(\mathbf{p}) \varepsilon_{v}^{(2)}(\mathbf{p})-\varepsilon_{v}^{(1)}(\mathbf{p}) \varepsilon_{\mu}^{(2)}(\mathbf{p})\right]$. On using explicit forms for the momentumspace polarization vectors we have:

$$
A^{\mu v}(\mathbf{p})=\frac{i N^{2}}{m}\left(\begin{array}{cccc}
0 & -p_{2} & p_{1} & 0 \\
p_{2} & 0 & m+\frac{p_{r} p_{l}}{p_{0}+m} & \frac{p_{2} p_{3}}{p_{0}+m} \\
-p_{1} & -m-\frac{p_{r} p_{l}}{p_{0}+m} & 0 & -\frac{p_{1} p_{3}}{p_{0}+m} \\
0 & -\frac{p_{2} p_{3}}{p_{0}+m} & \frac{p_{1} p_{3}}{p_{0}+m} & 0
\end{array}\right) .
$$

It coincides with the longitudinal components of the antisymmetric tensor obtained in Refs. [1, Eqs. $(2.14,2.17)]$ and $[14$, Eqs.(17b,18b)] within the normalization and different choice of the spin basis. We agree with the previous authors, e. g., Ref. [21], see Eq. (4) therein, about the gauge noninvariance of the division of the angular momentum of the electromagnetic field into the "orbital" and "spin" part, Eq. (19). We proved again that for the antisymmetric tensor field $\mathbf{J} \sim \int d^{3} \mathbf{x}(\mathbf{E} \times \mathbf{A})$.

We can generalize the BW formalism expanding the symmetric spinor field in the following way:

$$
\Psi_{\{\alpha \beta\}}=\left(\gamma^{\mu} R\right)_{\alpha \beta}\left(c_{a} m A_{\mu}+c_{f} F_{\mu}\right)+\left(\sigma^{\mu v} R\right)_{\alpha \rho}\left(c_{A} m\left(\gamma^{5}\right)_{\rho \beta} A_{\mu v}+c_{F} I_{\rho \beta} F_{\mu v}\right) .
$$

The new Proca-like equations are:

$$
\begin{aligned}
& c_{a} m\left(\partial_{\mu} A_{v}-\partial_{v} A_{\mu}\right)+c_{f}\left(\partial_{\mu} F_{v}-\partial_{v} F_{\mu}\right)=i c_{A} m^{2} \varepsilon_{\alpha \beta \mu v} A^{\alpha \beta}+2 m c_{F} F_{\mu v}, \\
& c_{a} m^{2} A_{\mu}+c_{f} m F_{\mu}=i c_{A} m \varepsilon_{\mu v \alpha \beta} \partial^{v} A^{\alpha \beta}+2 c_{F} \partial^{v} F_{\mu v} .
\end{aligned}
$$

In the case $c_{a}=1, c_{F}=\frac{1}{2}$ and $c_{f}=c_{A}=0$ they are reduced to the ordinary Proca equations. In the general case we obtain dynamical equations which connect the photon, the notoph and their 
potentials. The divergent (in the $m \rightarrow 0$ limit) parts of field functions and those of dynamical variables should be removed by the corresponding gauge (or the Kalb-Ramond gauge) transformations. Apart from these dynamical equations we can obtain a number of constraints by means of the subtraction of the equations of the Bargmann-Wigner system (instead of the addition). They give $\widetilde{F}^{\mu v} \sim i m A^{\mu v}$ and $F^{\mu} \sim m A^{\mu}$. The limit $m \rightarrow 0$ may be taken for dynamical variables, in the end of calculations only. Moreover, it might be logical to introduce the normalization scalar field $\varphi(x)$ and consider the expansion $\Psi_{\{\alpha \beta\}}=\left(\gamma^{\mu} R\right)_{\alpha \beta}\left(\varphi A_{\mu}\right)+\left(\sigma^{\mu v} R\right)_{\alpha \beta} F_{\mu v}$. Then, we arrive at the set of equations, which in the case of the constant scalar field $\varphi=2 m$, can again be reduced to the system of the Proca equations.

The spin- 2 case can be treated in a similar way. Now we use the equations for the 4-rank symmetric spinor. We proceed expanding the field function in the complete set of symmetric matrices. In the beginning let us use the first two indices:

$$
\Psi_{\{\alpha \beta\} \gamma \delta}=\left(\gamma_{\mu} R\right)_{\alpha \beta} \Psi_{\gamma \delta}^{\mu}+\left(\sigma_{\mu \nu} R\right)_{\alpha \beta} \Psi_{\gamma \delta}^{\mu \nu} .
$$

Next, we present the vector-spinor and tensor-spinor functions as

$$
\begin{aligned}
& \Psi_{\{\gamma \delta\}}^{\mu}=\left(\gamma^{\kappa} R\right)_{\gamma \delta} G_{\kappa}{ }^{\mu}+\left(\sigma^{\kappa \tau} R\right)_{\gamma \delta} F_{\kappa \tau}^{\mu}, \\
& \Psi_{\{\gamma \delta\}}^{\mu v}=\left(\gamma^{\kappa} R\right)_{\gamma \delta} T_{\kappa}{ }^{\mu v}+\left(\sigma^{\kappa \tau} R\right)_{\gamma \delta} R_{\kappa \tau}^{\mu \nu},
\end{aligned}
$$

i. e., using the symmetric matrix coefficients in indices $\gamma$ and $\delta$. Hence, the resulting tensor equations coincide with the equations obtained in Ref. [22]. However, we need to make symmetrization over two sets of indices $\{\alpha \beta\}$ and $\{\gamma \delta\}$. The total symmetry can be ensured if one contracts the function $\Psi_{\{\alpha \beta\}\{\gamma \delta\}}$ with the antisymmetric matrices $R_{\beta \gamma}^{-1},\left(R^{-1} \gamma^{5}\right)_{\beta \gamma}$ and $\left(R^{-1} \gamma^{5} \gamma^{\lambda}\right)_{\beta \gamma}$, and equate all these contractions to zero. We obtain additional constraints on the tensor field functions.

Nevertheless, we should modify the formalism in the spirit of Ref. [15]. The field functions take now into account $\gamma^{5} \sigma^{\mu v} R$ terms. Hence, the function $\Psi_{\{\alpha \beta\}\{\gamma \delta\}}$ can be expressed as a sum of nine terms. The corresponding dynamical equations are given in the following form:

$$
\begin{aligned}
& \frac{2 \alpha_{2} \beta_{4}}{m} \partial_{v} T_{\kappa}{ }^{\mu v}+\frac{i \alpha_{3} \beta_{7}}{m} \varepsilon^{\mu v \alpha \beta} \partial_{v} \widetilde{T}_{\kappa, \alpha \beta}=\alpha_{1} \beta_{1} G_{\kappa}{ }^{\mu}, \\
& \frac{2 \alpha_{2} \beta_{5}}{m} \partial_{v} R_{\kappa \tau}{ }^{\mu v}+\frac{i \alpha_{2} \beta_{6}}{m} \varepsilon_{\alpha \beta \kappa \tau} \partial_{v} \widetilde{R}^{\alpha \beta, \mu v}+\frac{i \alpha_{3} \beta_{8}}{m} \varepsilon^{\mu v \alpha \beta} \partial_{v} \widetilde{D}_{\kappa \tau, \alpha \beta}- \\
- & \frac{\alpha_{3} \beta_{9}}{2} \varepsilon^{\mu v \alpha \beta} \varepsilon_{\lambda \delta \kappa \tau} D_{\alpha \beta}^{\lambda \delta}=\alpha_{1} \beta_{2} F_{\kappa \tau}{ }^{\mu}+\frac{i \alpha_{1} \beta_{3}}{2} \varepsilon_{\alpha \beta \kappa \tau} \widetilde{F}^{\alpha \beta, \mu}, \\
& 2 \alpha_{2} \beta_{4} T_{\kappa}{ }^{\mu v}+i \alpha_{3} \beta_{7} \varepsilon^{\alpha \beta \mu v} \widetilde{T}_{\kappa, \alpha \beta}=\frac{\alpha_{1} \beta_{1}}{m}\left(\partial^{\mu} G_{\kappa}{ }^{v}-\partial^{v} G_{\kappa}{ }^{\mu}\right), \\
& 2 \alpha_{2} \beta_{5} R_{\kappa \tau}{ }^{\mu v}+i \alpha_{3} \beta_{8} \varepsilon^{\alpha \beta \mu v} \widetilde{D}_{\kappa \tau, \alpha \beta}+i \alpha_{2} \beta_{6} \varepsilon_{\alpha \beta \kappa \tau} \widetilde{R}^{\alpha \beta, \mu v}- \\
& -\frac{\alpha_{3} \beta_{9}}{2} \varepsilon^{\alpha \beta \mu v} \varepsilon_{\lambda \delta \kappa \tau} D^{\lambda \delta}=\frac{\alpha_{1} \beta_{2}}{m}\left(\partial^{\mu} F_{\kappa \tau}{ }^{v}-\partial^{v} F_{\kappa \tau}{ }^{\mu}\right)+ \\
& +\frac{i \alpha_{1} \beta_{3}}{2 m} \varepsilon_{\alpha \beta \kappa \tau}\left(\partial^{\mu} \widetilde{F}^{\alpha \beta, v}-\partial^{v} \widetilde{F}^{\alpha \beta, \mu}\right) .
\end{aligned}
$$

In general, the coefficients $\alpha_{i}$ and $\beta_{i}$ may now carry some dimension. The essential constraints can be found in Ref. [23]. They are the results of contractions of the field function with six antisymmetric matrices as above. The certain combinations of field functions can be equal to zero. The 
fields $F_{\kappa \tau}{ }^{\mu}, \widetilde{F}_{\kappa \tau}{ }^{\mu}, T_{\kappa}{ }^{\mu \nu}, \widetilde{T}_{\kappa}{ }^{\mu \nu}$, and $R_{\kappa \tau}{ }^{\mu \nu}, \widetilde{R}_{\kappa \tau}{ }^{\mu \nu}, D_{\kappa \tau}{ }^{\mu \nu}, \widetilde{D}_{\kappa \tau}{ }^{\mu \nu}$ can correspond to different physical states and the equations describe couplings one state to another.

Furthermore, from the set of equations (27-30) one obtains the second-order equation for the symmetric traceless tensor of the second rank $\left(\alpha_{1} \neq 0, \beta_{1} \neq 0\right)$ :

$$
\frac{1}{m^{2}}\left[\partial_{v} \partial^{\mu} G_{\kappa}{ }^{v}-\partial_{\nu} \partial^{v} G_{\kappa}^{\mu}\right]=G_{\kappa}^{\mu}
$$

After the contraction in indices $\kappa$ and $\mu$ this equation is reduced to:

$$
\partial_{\alpha} G_{\beta}^{\alpha}=F_{\beta}, \quad \frac{1}{m^{2}} \partial_{\beta} F^{\beta}=0,
$$

i. e., the equations which connect the analogue of the energy-momentum tensor and the analogue of the 4-vector field.

The possibility of terms such as $\sigma \cdot\left[\mathbf{A} \times \mathbf{A}^{*}\right]$ appears to be related to the matters of chiral interactions [24, 25]. The Dirac field operator can always be presented as a superposition of the self- and anti-self charge conjugate field operators. The anti-self charge conjugate part can give the self-charge conjugate part after multiplying by the $\gamma^{5}$ matrix, and vice versa. We derived

$$
\begin{aligned}
& {\left[i \gamma^{\mu} D_{\mu}^{*}-m\right] \psi_{1}^{s}=0,} \\
& {\left[i \gamma^{\mu} D_{\mu}-m\right] \psi_{2}^{a}=0 .}
\end{aligned}
$$

Both equations lead to the terms of interaction such as $\sigma \cdot\left[\mathbf{A} \times \mathbf{A}^{*}\right]$ provided that the 4-vector potential is considered as a complex function(al). In fact, from (33) we have:

$$
i \sigma^{\mu} \nabla_{\mu} \chi_{1}-m \phi_{1}=0, \quad i \tilde{\sigma}^{\mu} \nabla_{\mu}^{*} \phi_{1}-m \chi_{1}=0 .
$$

And, from (34) we have

$$
i \sigma^{\mu} \nabla_{\mu}^{*} \chi_{2}-m \phi_{2}=0, \quad i \tilde{\sigma}^{\mu} \nabla_{\mu} \phi_{2}-m \chi_{2}=0 .
$$

The meanings of $\sigma^{\mu}$ and $\tilde{\sigma}^{\mu}$ are obvious from the definition of $\gamma$ matrices. The derivatives are defined $D_{\mu}=\partial_{\mu}-i e \gamma^{5} C_{\mu}+e B_{\mu}, \quad \nabla_{\mu}=\partial_{\mu}-i e A_{\mu}$, and $A_{\mu}=C_{\mu}+i B_{\mu}$. Thus, relations with the magnetic monopoles can also be studied. From the above systems we extract the terms as $\pm e^{2} \sigma^{i} \sigma^{j} A_{i} A_{j}^{*}$, which lead to the discussed terms [24, 25]. We would like to note that the terms of the type $\sigma \cdot\left[\mathbf{A} \times \mathbf{A}^{*}\right]$ can be reduced to $(\sigma \cdot \nabla) V$, where $V$ is the scalar potential.

The most general relativistic-invariant Lagrangian for the symmetric 2nd-rank tensor is

$$
\begin{aligned}
& L=-\alpha_{1}\left(\partial^{\alpha} G_{\alpha \lambda}\right)\left(\partial_{\beta} G^{\beta \lambda}\right)-\alpha_{2}\left(\partial_{\alpha} G^{\beta \lambda}\right)\left(\partial^{\alpha} G_{\beta \lambda}\right)- \\
& -\alpha_{3}\left(\partial^{\alpha} G^{\beta \lambda}\right)\left(\partial_{\beta} G_{\alpha \lambda}\right)+m^{2} G_{\alpha \beta} G^{\alpha \beta} .
\end{aligned}
$$

It leads to the equation

$$
\left[\alpha_{2}\left(\partial_{\alpha} \partial^{\alpha}\right)+m^{2}\right] G^{\{\mu v\}}+\left(\alpha_{1}+\alpha_{3}\right) \partial^{\{\mu \mid}\left(\partial_{\alpha} G^{\alpha \mid v\}}\right)=0 .
$$

In the case $\alpha_{2}=1>0$ and $\alpha_{1}+\alpha_{3}=-1$ it coincides with Eq. (31). There is no any problem to obtain the dynamical invariants for the fields of the spin 2 from the above Lagrangian. The mass 
dimension of $G^{\mu v}$ is [energy $]^{1}$. We now present possible relativistic interactions of the symemtric 2-rank tensor. The simplest ones should be the the following ones: $L_{(1)}^{i n t} \sim G_{\mu v} F^{\mu} F^{v}, L_{(2)}^{i n t} \sim$ $\left(\partial^{\mu} G_{\mu v}\right) F^{v}, L_{(3)}^{\text {int }} \sim G_{\mu v}\left(\partial^{\mu} F^{v}\right)$.

It is also interesting to note that thanks to the possible terms

$$
V(F)=\lambda_{1}\left(F_{\mu} F^{\mu}\right)+\lambda_{2}\left(F_{\mu} F^{\mu}\right)\left(F_{v} F^{v}\right)
$$

we can give the mass to the tensor components of the spin-2 field. This is due to the possibility of the Higgs-like spontaneous symmetry breaking, with $v$ being the vacuum expectation value, $v^{2}=\left(F_{\mu} F^{\mu}\right)=-\lambda_{1} / 2 \lambda_{2}>0$. Several degrees of freedom of the 4-vector field are removed. An arbitrary phase for $F^{\mu}$ is only possible if the 4-vector would be the complex one. The similar conslusion can be done if we would use the AST field of the 2nd rank or the $(1,0) \oplus(0,1)$ field as in [26]. However, the vacuum expectation value (VEV), of course, may be different. So, the interplay between the VEV's, the dynamical equations, normalization (and so on) deserves further investigation on the experimental/observational level. ${ }^{1}$

Next, since the interaction of fermions with notoph, for instance, can be considered as that of the order $\sim e^{2}$ in the initial Lagrangian, it is more difficult to observe it. However, as far as I know the theoretical precision calculus in QED (the Landé factor, the anomalous magnetic moment, the hyperfine splittings in positronium and muonium, and the decay rates of $o-P s$ and $p-P s$ ) are near the order corresponding to the 4th-5th loops, where the difference may appear with the experiments, cf. [27].

We considered the Bargmann-Wigner formalism in order to derive the equations for the AST fields, and for the symmetric tensor of the 2nd rank. We introduced the additional normalization scalar field in the Bargmann-Wigner formalism in order to account for possible physical significance of the Ogievetskii-Polubarinov-Kalb-Ramond modes. Both the antisymmetric tensor fields and the 4-vector fields may have the third helicity state in the massless limits. This problem is connected with the problem of the observability of the gauge [20]. We introduced the additional symmetric matrix in the Bargmann-Wigner expansion $\left(\gamma^{5} \sigma^{\mu v} R\right)$ in order to account for the dual fields. The problem was discussed, what are the the correct definitions of the energy-momentum tensor and other Nöther currents in the electromagnetic theory, the relativistic theory of gravitation, the general relativity, and their generalizations. The notoph-graviton interaction may give the mass to spin-2 particles in the way similar to the spontaneous-symmetry-breaking Higgs formalism [28]. I acknowledge discussions with participants of recent conferences on Fundamental Physics, Symmetries and Clifford Algebras.

\section{References}

[1] D. V. Ahluwalia and D. J. Ernst, Int. J. Mod. Phys. E2 (1993) 397.

[2] D. V. Ahluwalia and M. Sawicki, Phys. Rev. D47 (1993) 5161.

[3] V. V. Dvoeglazov, Int. J. Theor. Phys. 37 (1998), 1915; hep-th/9611068.

[4] V. V. Dvoeglazov, Hadronic J. Suppl. 12 (1997) 221.

\footnotetext{
${ }^{1}$ I would still be cautious to use the second-order derivative in the Lagrangian as opposed to [26]. Particularly, in the theories where the 4-momentum is not defined in the unique way. See the 19th century Ostrogradsky papers.
} 
[5] S. Weinberg, Phys. Rev. B133 (1964) 1318; ibid. B134 (1964) 882; ibid. 181 (1969) 1893.

[6] R. H. Tucker and C. L. Hammer, Phys. Rev. D3 (1971) 2448.

[7] V. I. Ogievetskiĭ and I. V. Polubarinov, Yadern. Fiz. 4 (1966) 216 [English translation: Sov. J. Nucl. Phys. 4 (1967) 156].

[8] K. Hayashi, Phys. Lett. B44 (1973) 497.

[9] M. Kalb and P. Ramond, Phys. Rev. D9 (1974) 2273.

[10] E. Durandin and A. Erschow, Phys. Z. Sowjet. 12 (1937) 466.

[11] F. Belinfante, Physica 6 (1939) 887.

[12] L. V. Avdeev and M. V. Chizhov, Phys. Lett. B321 (1994) 212; hep-th/9407067.

[13] M. V. Chizhov, Phys. Part. Nucl. 42 (2011) 93.

[14] V. V. Dvoeglazov, Czech. J. Phys. 50 (2000) 225, hep-th/9712036.

[15] V. V. Dvoeglazov, Phys. Scripta 64 (2001) 201, physics/9804010.

[16] V. Bargmann and E. P. Wigner, Proc. Natl. Acad. Sci. (USA) 34 (1948) 211.

[17] D. Luriè, Particles and Fields (Interscience Publishers, 1968). Ch. 1.

[18] M. Kirchbach, Mod. Phys. Lett. A12 (1997) 2373.

[19] S. Weinberg, The Quantum Theory of Fields. Vol. I. Foundations. (Cambridge University Press, 1995), Ch. 5.

[20] A. Staruszkiewicz, Acta Phys. Polon. B13 (1982) 617; ibid. 14 (1983) 63, 67, 903; ibid. 15 (1984) 225; ibid 23 (1992) 591.

[21] H. C. Ohanian, Am. J. Phys. 54 (1986) 500.

[22] G. Marques and D. Spehler, Mod. Phys. Lett. A13 (1998) 553.

[23] V. V. Dvoeglazov, Adv. Appl. Clifford Algebras 10 (2000) 7, math-ph/9805017.

[24] V. V. Dvoeglazov, Electromagnetic Phenomena, 2, No. 1 (5) (2001) 3, hep-ph/9801287.

[25] V. V. Dvoeglazov, Acta Phys. Slovaca 50 (2000) 629.

[26] D. V. Ahluwalia an T. Goldman, Mod. Phys. Lett. A8 (1993) 2623, hep-ph/9304242.

[27] V. V. Dvoeglazov, R. N. Faustov and Yu. N. Tyukhtyaev, Phys. Part. Nucl. 25 (1994) 58.

[28] V. V. Dvoeglazov, Int. J. Mod. Phys. CS 3 (2011) 121. 